\title{
The fluid-thermal-solid coupling analysis of a scroll expander used in an ORC waste heat recovery system
}

Zhen Liu ${ }^{1,4}$, Mingshan Wei ${ }^{1,}{ }^{*}$, Panpan Song ${ }^{2}$, Simon Emhardt ${ }^{3}$, Guohong Tian ${ }^{3}$, Zhi Huang ${ }^{1}$

1. School of Mechanical Engineering, Beijing Institute of Technology, Beijing 100081, China

2. State Key Laboratory of Automotive Safety and Energy, Tsinghua University, Beijing 100084, China

3. Department of Mechanical Engineering Sciences, University of Surrey, Guildford, GU2 7HX, UK

4. School of Mechanical and Automotive Engineering, Hubei University of Arts and Science, Xiangyang, 441053, Hubei, China

\section{(*) Corresponding Author:}

Prof. Dr. Mingshan Wei

Address: School of Mechanical Engineering, Beijing Institute of Technology,

No. 5, Zhongguancun South Street, Haidian District, Beijing 100081

Tel: +861068911373

Fax: +861068912519

E-mail: mswei@bit.edu.cn

\section{Abstract}

In this research, a one-way fluid-thermal-solid numerical coupling model of a scroll 
expander for a waste heat recovery system was developed and used to investigate the deformation of the scroll pair. The pressure and thermal loads were firstly calculated by a CFD model, and the surface pressure and body temperature distributions of the scroll members were used as boundary conditions in the FEM model to obtain the deformation distributions of the scroll parts. Three time instants that may have significant adverse impacts on the maximum forces were selected to determine the most critical time for the occurrence of the maximum deformation of the scroll wraps. The results showed that the deformations induced by inertial force only occurred at the orbiting scroll tail, whereas the deformations in other regions negligible. At the time instants of $t / T$ equaled to $13 / 15$ and 1 , the deformations induced by pressure loads had the opposite direction compared to that of the thermal loads and thus the two deformations canceled each other out and the coupling deformations decreased. The deformations induced by pressure loads were less significant than the thermal loads, therefore the coupling deformation was dominated by the thermal loads. The results also confirmed that the critical time at $t / T$ equaled to $7 / 20$ for the occurrence of the largest deformation resulted from the maximum axial forces that were exerted on the fixed scroll.

\section{Keywords}

Scroll expander; Fluid-thermal-solid coupling; Deformation analysis; Pressure and thermal loads

\section{Introduction}

In recent years, scroll expanders have gained increasing interest as the expansion machine of organic Rankine cycle (ORC) based Waste Heat Recovery (WHR) systems. As indicated in Ref.[1, 2], the scroll expander is more suitable than the turbine expander for small-scale ORC 
systems, i.e. power output below $10 \mathrm{~kW}$. Moreover, the scroll expander has advantages over other positive displacement expanders for the ORC application, due to its less moving components, higher efficiency, and lower cost $[3,4]$. Nevertheless, the manufacturing tolerances and clearances between the mating scrolls are a vital design consideration of scroll expanders, as larger clearances can heavily affect their performance. Oversized clearances will lead to increased leakages, while wear and tear caused by friction or even jamming of the scrolls potentially occur when the clearances are excessively tight. The deformations of the scroll wraps and endplates should be therefore investigated in order to determine appropriate design clearances. Up to date, there are a few published studies on deformation analysis of scroll machines. Numerical investigations by employing Finite Element Methods (FEM) were carried out to examine the deformation of the mating scrolls of scroll compressors, in order to provide a solid reference for the scroll expander development. Kazutaka Suefuji et al. [5] assessed for the first time that the main reasons for the deformation were the pressure and thermal loads acting upon the scroll wraps. Yu et al. [6] applied heat transfer boundary conditions on scroll wrap surfaces and assigned the uniform pressure along the axial direction to each symmetrical chamber pairs to employ an FEM model for the capture of the fixed scroll deformation. O'Leary et al. [7] created an FEM model in order to yield the deformation distribution of scroll parts based on obtained pressure and thermal loads determined through a thermodynamic simulation model. Lin et al. [8] used thermocouples to measure the temperature in specified test points in a fixed scroll plate, and combined the temperature measurements with an FEM model for the analysis of the thermal deformation and stress distribution of the scrolls. Liu et al. [9] carried out the finite element analysis for different 
geometric parameters of the orbiting scrolls to determine the optimum design of the scrolls by utilising the lower deformations, which also obtained pressure and thermal loads through a thermodynamic simulation model.

According to previous CFD modelling results of scroll machines, the pressure and temperature distributions inside the working chambers were uneven [4,10-16] and the existing empirical formulas to predict of the convective heat transfer coefficient were not suitable for the compression process [12]. However, these previous studies on the deformation analysis were generally on the linear loading or used the empirical formulas to determine the boundary conditions. Furthermore, the heating effect of the gas in the plenum was not considered. In addition, the deformation distribution was only calculated and analysed at the beginning of the compression process, which was considered as the most detrimental time instant. Compared to a scroll compressor, the pressure and temperature distributions in a scroll expander are different due to the reversed working process [4], which will certainly lead to a different deformation behaviour of the scroll parts. For this reason, the most critical time instant on scroll expander deformations need further investigations.

In this work, an improved methodology was developed, in which the CFD simulation results were integrated into an FEM model as boundary conditions. The evaluation of the transient working process in the scroll chamber and the temperature variations of the scroll wraps and endplates was determined by the CFD model. Subsequently, the calculated results of surface pressure and body temperature on the mating scrolls were used to define the boundary conditions for an FEM model employing one-way coupling method, in which only the effects of gas flow on the pressure and temperature distributions on the walls of scroll 
wrap were considered. However, the influence of the structural displacements on the gas flow field was not taken into account. The deformation distributions of the scroll pair were determined at different times. Moreover, the deformation results at the critical time when the maximum deformation occurred were analysed.

\section{Model and methodology}

\subsection{Physical model}

The specific geometric parameters and material properties of the scroll plates are summarized in Table 1 . The primary geometry parameters of the scroll wraps are the same as that in Ref. [15]. The material properties used in fluid-thermal-solid coupling analysis are the same as those of real scroll parts, and the real scroll parts are made of Aluminum alloy $4032[17,18]$

Table 1 Geometric parameters and material properties of the scroll plates.

\begin{tabular}{|c|c|c|c|}
\hline Parameter & Value & Property & Value \\
\hline Radius of basic circle $[\mathrm{mm}]$ & 3.2 & Elasticity modulus [GPa] & 79 \\
\hline Inner involute initial angle [rad] & 0.698 & Poisson ratio[-] & 0.33 \\
\hline Outer involute initial angle [rad] & -0.698 & Density $\left[\mathrm{kg} / \mathrm{cm}^{3}\right]$ & 2680 \\
\hline Involute ending angle [rad] & 17.08 & Fatigue strength[MPa] & 315 \\
\hline Thickness of endplate[mm] & 8 & Tensile strength[MPa] & 380 \\
\hline Height of suction plenum[mm] & 12 & Specific heat $[\mathrm{J} /(\mathrm{kg} \cdot \mathrm{K})]$ & 864 \\
\hline Orbiting radius $[\mathrm{mm}]$ & 5.58 & Coefficient of expansion $\left[\mathrm{K}^{-1}\right]$ & $1.95 \mathrm{e}-05$ \\
\hline Inner involute starting angle[rad] & 5.759 & Heat transfer coefficient $[\mathrm{W} /(\mathrm{m} \cdot \mathrm{K})]$ & 141 \\
\hline Outer involute starting angle [rad] & 2.617 & & \\
\hline Height of scroll wrap [mm] & 33 & & \\
\hline Outer diameter of endplate $[\mathrm{mm}]$ & 116 & & \\
\hline
\end{tabular}

The structure of the scroll expander used in the numerical simulation and testing is shown in Fig. 1. In particular, the machine was converted from an open-drive automobile air conditioning compressor. When the scroll compressor is operated as ORC expander, continuous gases at high pressure and temperature enter the suction plenum that in turn heat 
the cool walls and exert pressure on the endplate of the fixed scroll. Due to the ever-changing shape of each gas pocket in the working chambers, an unsteady flow field distribution is generated causing a constantly changing stress and deformation of the scroll solid.

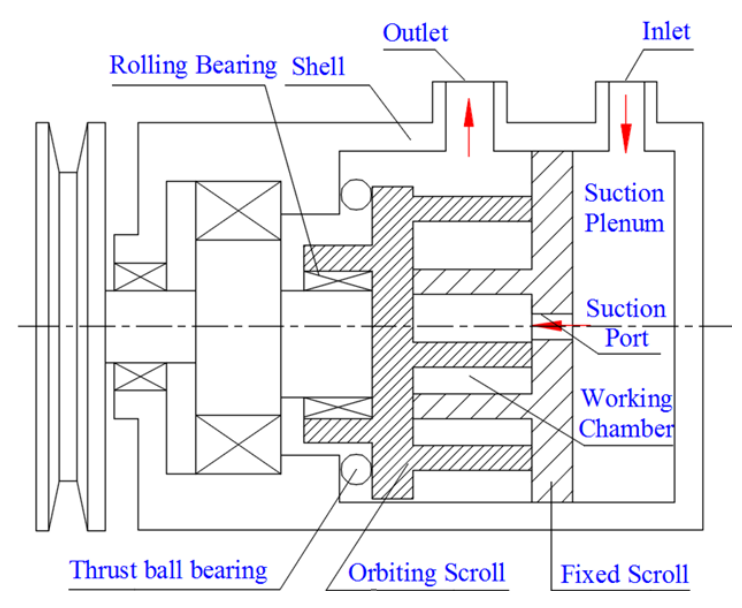

Fig. 1 Structure schematic drawing of scroll expander.

\subsection{CFD model and experimental validation}

The commercial code ANSYS FLUENT 17.0 was used for this analysis. Though 2D CFD simulation can handle radial clearances as low as $20 \mu \mathrm{m}$ [4], the gas force and heating effect of the gas in the suction plenum can't be reflected in the 2D numerical models. In order to obtain the accurate pressure and thermal loads, the CFD simulation adopted the fluid-thermal-solid coupling method in combination with a 3D transient numerical model using the dynamic mesh strategy. After trade-off between the computational time versus accuracy of results, the radial clearance was set to $200 \mu \mathrm{m}$ in the present work, and the axial clearance was not considered. Overall performances of the scroll machine are not influenced by the time step [4], and this analysis is more concerned about the gas force and temperature exerted on the scrolls other than local flow field such as leakage flow. The uniform angle-interval chosen, in accordance with [15], was set to $1.2 \mathrm{deg}$.

The computational domain of the model is shown in Fig.2. The fluid domain is composed 
of inlet, suction plenum, suction port, scroll working chambers and outlet. The solid domain is composed of the fixed and orbiting scroll wraps with endplates. The movement of the solid parts are mechanically constrained properly. Fig. 3 shows the grids of the computational domain. It consists of unstructured grids with 875,595 nodes and $1,636,687$ cells in the scroll working chambers, 52,670 nodes and 69,818 cells in the scroll wrap solid, and a structured grid with $1,156,891$ cells in the inlet, suction plenum, suction port, outlet and scroll plate solid.

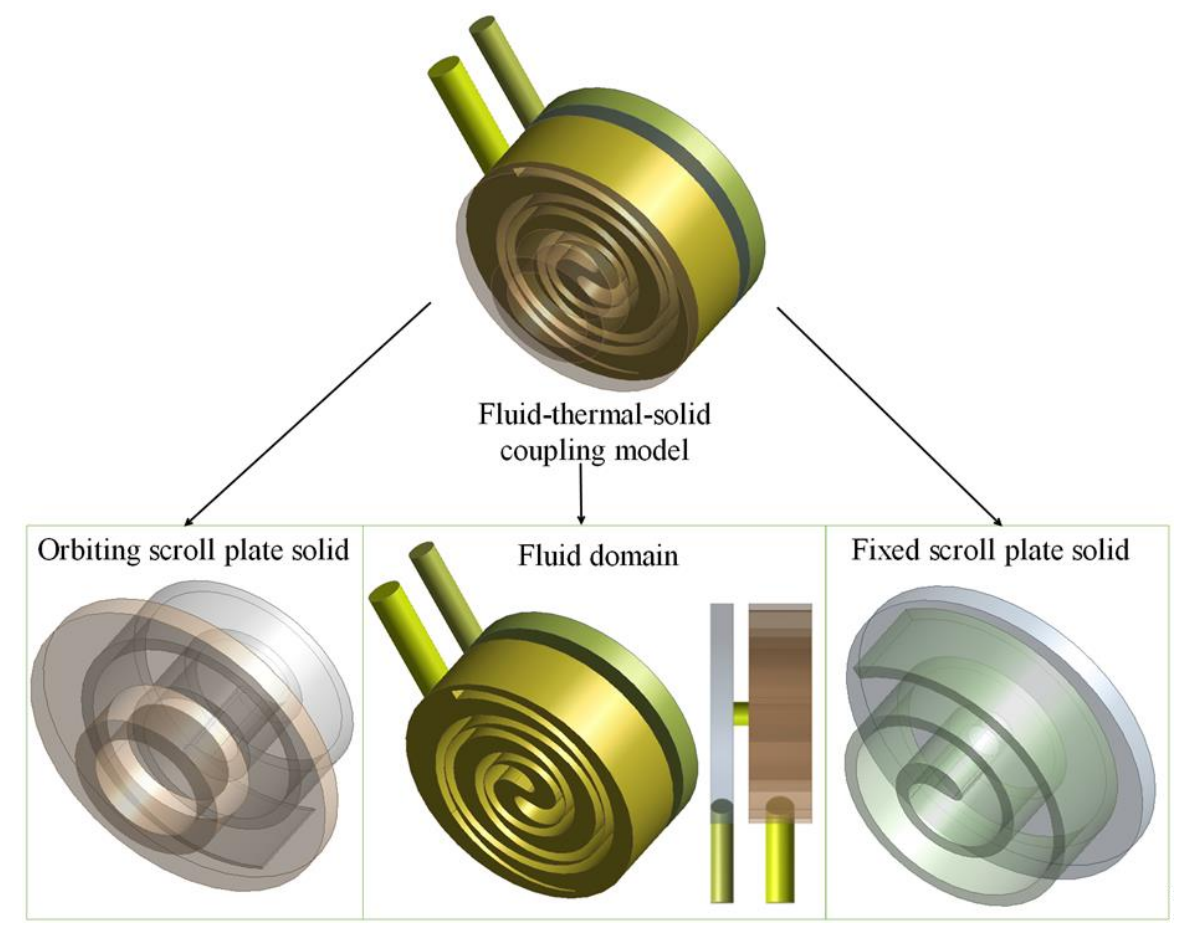

Fig. 2 Computational domains of the scroll expander.

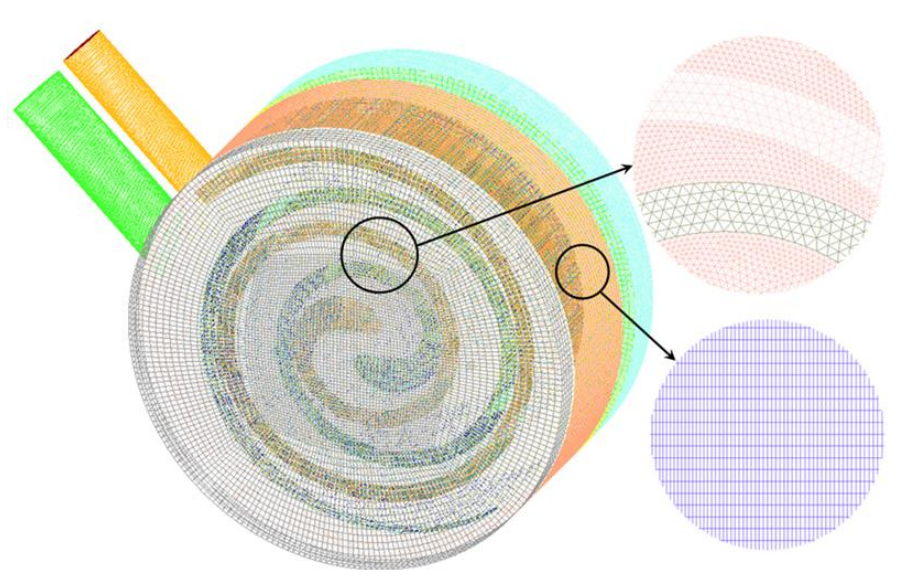

Fig. 3 Illustration of the grids for computational domain. 
In the current case, R123 was selected as the working fluid from the NIST real gas model. Coupled algorithm was selected as the pressure-velocity coupling scheme which solves the pressure-based and momentum continuity equations together. Gas flow in the internal scroll expander was governed by Reynolds Averaged Naiver-Stokes equations, where the k-epsilon model with standard wall function including viscous heating was used as the turbulence model. It is worth noting that the interfaces between gas and surfaces of scroll parts were defined as thermal coupled walls. Bidirectional heat transfer between the fluid and solid was calculated. The conservation requirement of gas heat flux and solid heat flux must be met, which is given by:

$$
q_{\mathrm{s}}=q_{\mathrm{f}}
$$

where $q_{\mathrm{s}}$ and $q_{\mathrm{f}}$ represent the heat flux of solid and fluid domain on the interface respectively, and they are calculated by Eqs. (2) and (3).

$$
\begin{gathered}
q_{\mathrm{s}}=-K_{e f f} \nabla T_{\mathrm{s}} \\
q_{\mathrm{f}}=h_{\mathrm{f}}\left(T_{\mathrm{s}}-T_{\mathrm{f}}\right)+q_{\mathrm{rad}}
\end{gathered}
$$

where $K_{\text {eff }}$ represents the thermal conductivity of the scrolls material, and $h_{\mathrm{f}}$ represents the enthalpy of the gases. The radiation heat transfer is neglected in the calculation, therefore $q_{\mathrm{rad}}$ equals to 0.

The conservation of energy equation in the solid domain is described by:

$$
\frac{\partial}{\partial t}\left(\rho_{\mathrm{s}} h_{\mathrm{s}}\right)+\nabla \cdot\left(\rho_{\mathrm{s}} \mathrm{r} h_{\mathrm{s}}\right)=\nabla \cdot\left(K_{e f f} \nabla T_{\mathrm{s}}\right)
$$

where $\rho_{\mathrm{s}}, h_{\mathrm{s}}$ and $\stackrel{\hat{v}}{\mathrm{r}}$ represent density, enthalpy and velocity of the solid, respectively.

Since the resulting deformation is less than $2 \%$ compared to the average scroll wrap thickness, the influence of the deformation on the flow field was not taken into account. To 
validate the accuracy of the model, experimental measurements were carried out under four different test conditions, which are listed in Table 2 . The test rig was already introduced in the previous work $[15,16]$.

Table 2 Test conditions.

\begin{tabular}{ccccc}
\hline Test condition & $n /\left(\mathrm{r} \cdot \mathrm{min}^{-1}\right)$ & $P_{i} / \mathrm{kPa}$ & $T_{i} / \mathrm{K}$ & $P_{o} / \mathrm{kPa}$ \\
\hline 1 & 1986 & 835 & 383.50 & 216 \\
2 & 2000 & 1000 & 405.28 & 340 \\
3 & 2005 & 995 & 429.23 & 315 \\
4 & 2010 & 861 & 449.22 & 233 \\
\hline
\end{tabular}

The comparisons between experimental and simulation results under four test conditions are shown in Fig.4. It can be seen that the results of experiment and simulation results are in good agreement with each other, and the deviations are around 5\%. The experimental and simulated power output results of the scroll expander can be calculated by Eqs. (5) and (6).

$$
\begin{gathered}
W_{\text {exp }}=\frac{U \cdot I}{\eta} \\
W_{\mathrm{si} \mathrm{m}}=\frac{2 \pi n}{60 T} \cdot \int_{t}^{t+T} M_{d}(t) d
\end{gathered}
$$

where $U$ and $I$ represent measured voltage and current, respectively; internal friction losses and external electro-mechanical losses are lumped into one single efficiency $\eta$ of $55 \%[20,21] ; n$ is the rotating speed of the orbiting scroll; $M_{d}$ represents instantaneous driving gas moment exerted on the orbiting scroll. 


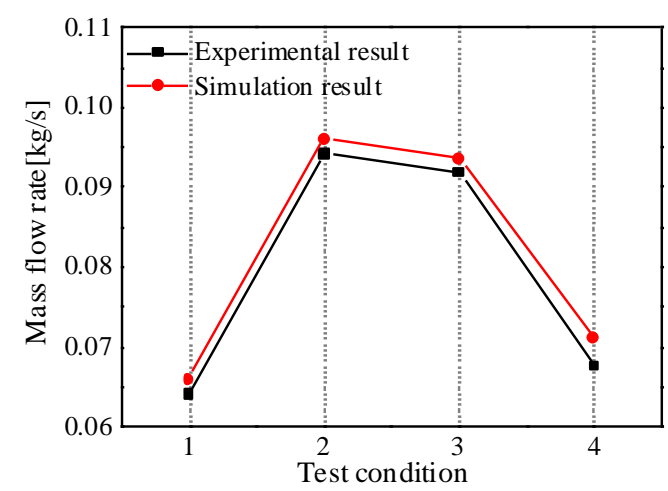

(a)

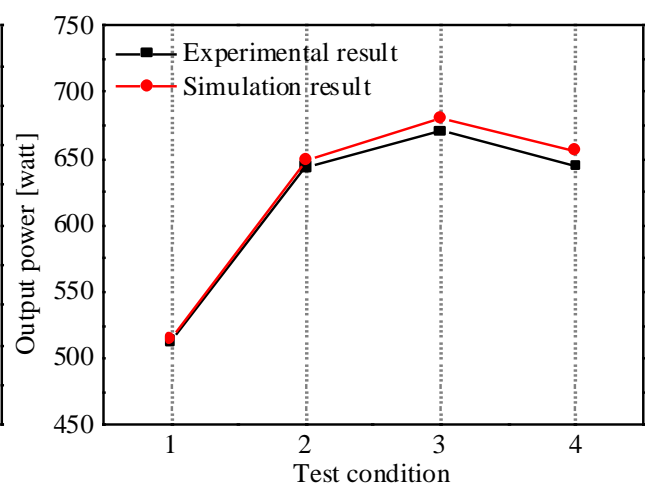

(b)

Fig. 4 Comparisons between experimental and simulation results: (a) mass flow rate and (b) output

power

\subsection{FEM model and loads of the scroll expander structure}

The results of pressure and temperature calculated from the CFD simulation were used as boundary conditions of the FEM model, and the calculation of deformation was performed in ANSYS Mechanical 17.0. The data transmission between the CFD and the FEM was carried out by means of a one-way coupling method. Several constrains were applied to the deformation analysis conforming to the real situation of the scroll expander. The constraints for the scroll parts are as follows:

(1) the degree-of-freedom in the z-axis direction at the position where the back of the endplate of the orbiting scroll that attaches to the anti-rotation mechanism by the thrust ball bearing is constrained;

(2) the degree-of-freedoms in the $\mathrm{x}$ - and $\mathrm{y}$-axis directions on the inside wall of the bearing hole that is used to mount the radial bearing parts are constrained;

(3) the degree-of-freedom in the $\mathrm{z}$ axis direction on the top wall of the bearing hole is constrained;

(4) the constrain condition of the fixed scroll is that the contact surface between the frame 
and fixed scroll plate is set as a fixed support.

During the working process, scroll expander is subjected to complex load conditions. These include the internal pressure from the gases, the thermal stresses, and the inertia forces. The contact stress between the mating parts was not considered. Furthermore, one of the main objectives of the deformation analysis was to determine the time instant when the largest deformation occurs, thereby characterized as the most detrimental time, and provide a reference for future researches to eliminate the contact between the mating scrolls. The main loads of the scroll expander involved in the deformation calculation are presented as follows:

(1) Pressure loads. As mentioned in [19], the exerted force on the orbiting scroll can be divided into forces acting in three different directions as shown in Fig.5. The three component forces are calculated by the CFD model using the following equations:

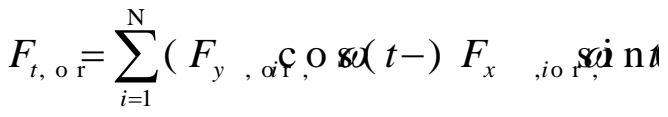

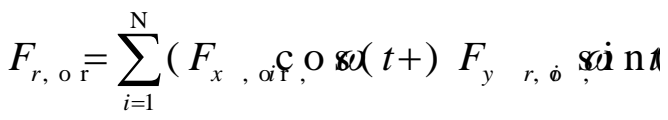

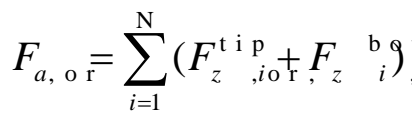

where $F_{t, \text { or }}$ is the tangential force acting on the orbiting scroll, which generates the driving movement of the orbiting scroll; $F_{r \text {,or }}$ is the radial force acting on the orbiting scroll, which is responsible for the shift of the fixed and orbiting scroll to each other which in turn increases the radial clearance between the mating scrolls; $F_{a, \text { or }}$ is the axial force acting the orbiting scroll, which is normal to the surface of the scroll endplate and top wall acting on the scroll wrap; $\mathrm{N}$ is the count of cells; $F_{x, \mathrm{or}, i}, F_{y, \mathrm{or}, i}$ and $F_{z, \mathrm{or}, i}$ are the component forces acting on the orbiting scroll along the $\mathrm{x}, \mathrm{y}, \mathrm{z}$ axis direction, respectively. 


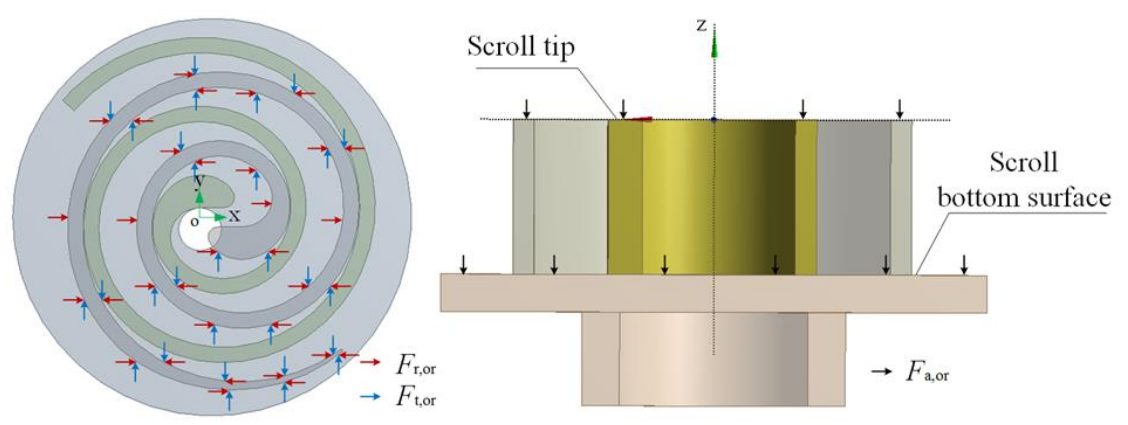

Fig. 5 Three component gas forces acting on the orbiting scroll.

In order to calculate the component forces acting on the fixed scroll, the fixed scroll was supposed to go through translational rotation around the center in the base circle of the orbiting scroll and the orbiting scroll was supposed to be fixed. The axial force acting on the fixed scroll includes the gas force from the working chamber and the suction plenum. The component forces of the fixed scroll are given by:

$$
\begin{aligned}
& F_{t, \mathrm{fix}}=\sum_{i=1}^{\mathrm{N}}\left(F_{y, \mathrm{fix}, i} \cos (\omega t)-F_{x, \mathrm{fix}, i} \sin (\omega t)\right)
\end{aligned}
$$

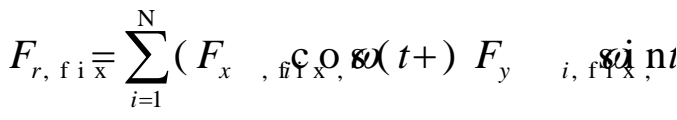

$$
\begin{aligned}
& F_{a, \text { fix }}=\sum_{\mathrm{i}=1}^{\mathrm{N}}\left(F_{z, \mathrm{fix}, i}^{\mathrm{tip}}+F_{z, \mathrm{fix}, i}^{\mathrm{bot}}+F_{z, \mathrm{ple}, i}^{\mathrm{top}}\right)
\end{aligned}
$$

where $F_{r}$ fix is the radial force acting on the fixed scrolls; $F_{t, f i x}$ is the tangential force acting on the fixed scroll; $F_{a, \text { fix }}$ is the axial force acting on the fixed scroll. $F_{x, \text { fix }, i}, F_{y, \text { fix }, i}$ and $F_{z, \text { fix }, i}$ are the component forces exerted on the orbiting scroll along the $\mathrm{x}-, \mathrm{y}-, \mathrm{z}$-axis direction, respectively.

(2) Thermal loads. The scroll expander used in the ORC system is always exposed to high temperatures. Thermal deformation occurs on the scroll solid due to the high expansion coefficient of aluminum alloy and the thermal stress is caused by the un-even temperature distribution. 
(3) Inertial loads. The inertial load of the orbiting scroll is caused by the constant translational rotation. The centrifugal acceleration generates inertial loads which are given by:

$$
F_{o c}=m_{o} R_{\mathrm{or}}(2 \pi n / 60)^{2}
$$

where $F_{o c}, m_{o}$, and $R_{\text {or }}$ are the centrifugal force, mass of the orbiting scroll and orbiting radius of the orbiting scroll, respectively.

\section{Flow distributions in the scroll expander}

\subsection{Gas forces exerted on the scroll parts}

The data in Table 3 lists the operation conditions for the scroll expander during the simulation.

Table 3 Simulated operating conditions.

\begin{tabular}{cccc}
\hline$P_{i} / \mathrm{kPa}$ & $T_{i} / \mathrm{K}$ & $P_{o} / \mathrm{kPa}$ & $n /\left(\mathrm{r} \cdot \mathrm{min}^{-1}\right)$ \\
\hline 1000 & 405 & 340 & 2000 \\
\hline
\end{tabular}

As mentioned in section 2.3, the forces are only applied against the lateral walls of the scroll wraps if pressure differences between the inner and outer parts of the scroll wraps exist. The axial forces are perpendicular to the surfaces of the scroll endplates and the values decrease gradually from the central suction chamber to the outmost backpressure chamber. Fig.6 shows the transient radial force $F_{r}$, tangential force $F_{t}$ and axial force $F_{a}$ acting on the fixed and orbiting scroll, which are calculated by employing the equations reported in section 2.3 and it can be seen that they fluctuate periodically. In Fig.6(a), the tangential and radial component forces acting on of the fixed and orbiting scrolls are in the same order of magnitude but acting in the opposite direction. When $F_{r}$, or reaches its peak value at $t / T=1$ (marked number 1), $F_{r}$, fix also reaches its maximum negative value at the same time. At point $t / T=13 / 15$ (marked as number 2) $F_{t, \text { fix }}$ reaches the maximum negative value, and $F_{t, \text { or }}$ achieves 
the peak value which indicates that the gas driving force acting upon the orbiting scroll and the pressure difference between the adjacent chambers both are the highest at this instant. The axial force exerted on the fixed endplate is much higher than that on the orbiting endplate as shown in Fig.6(b), and their peak values are marked as number 3 at $t / T=7 / 20$ and number 4 at $t / T=1$, respectively.

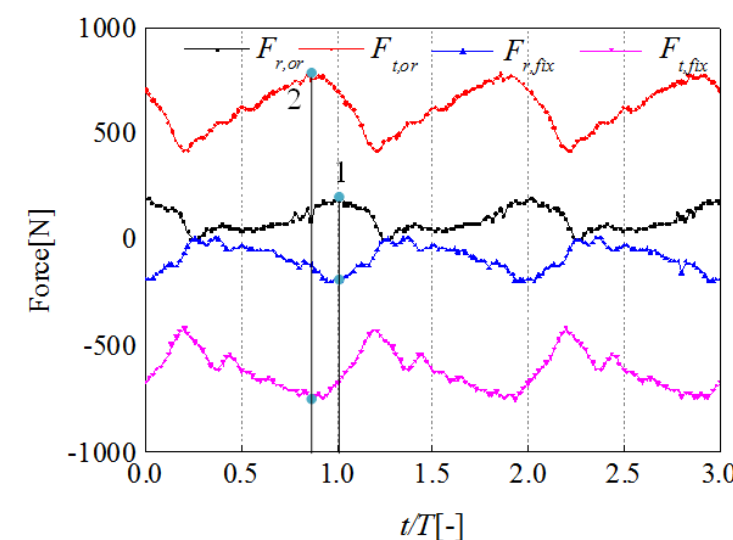

(a)

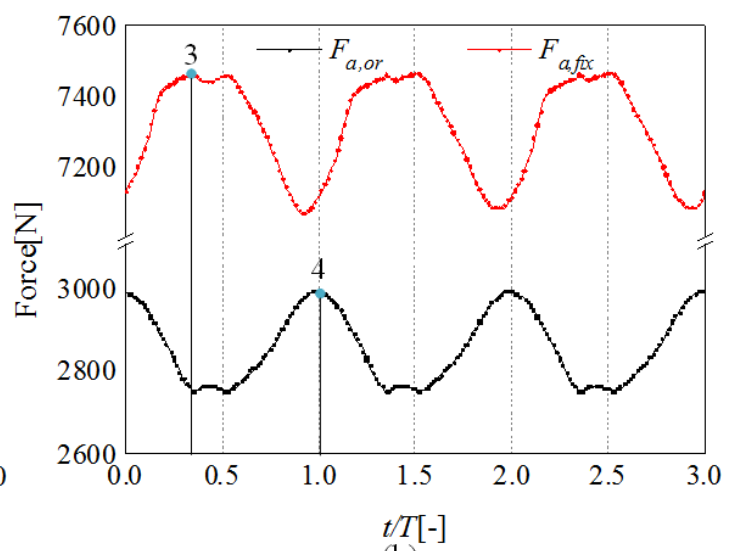

(b)

Fig. 6 Transient gas forces acting on the fixed and orbiting scroll:(a) $F_{t}$ and $F_{r}$, and (b) $F_{a}$.

3.2 Pressure differences between the inside and outside walls of the scroll wraps

The scroll expander working chambers were divided into several sub-chambers according to the suction, expansion and discharge processes as illustrated in Fig.7. The suction chamber (marked as Suc) comprises the chambers Suc1 and Suc2 at the beginning of the working process. The chamber Suc3 begins to form with the movement of the orbiting scroll. As a result, the chamber Suc1 is divided into Suc3 and Suc4 and the chamber Suc2 is divided into Suc3 and Suc5. The working fluid leaves the suction chambers after one revolution of the orbiting scroll and enters the expansion chambers which are divided into the chambers Exp1 and Exp2, respectively. The pair of exhaust chambers Exh1 and Exh2 are located next to the discharging ports, which are labeled with black dashed lines in Fig. 7. The outermost areas are the backpressure chambers and are marked as Bac1 and Bac2, respectively. 


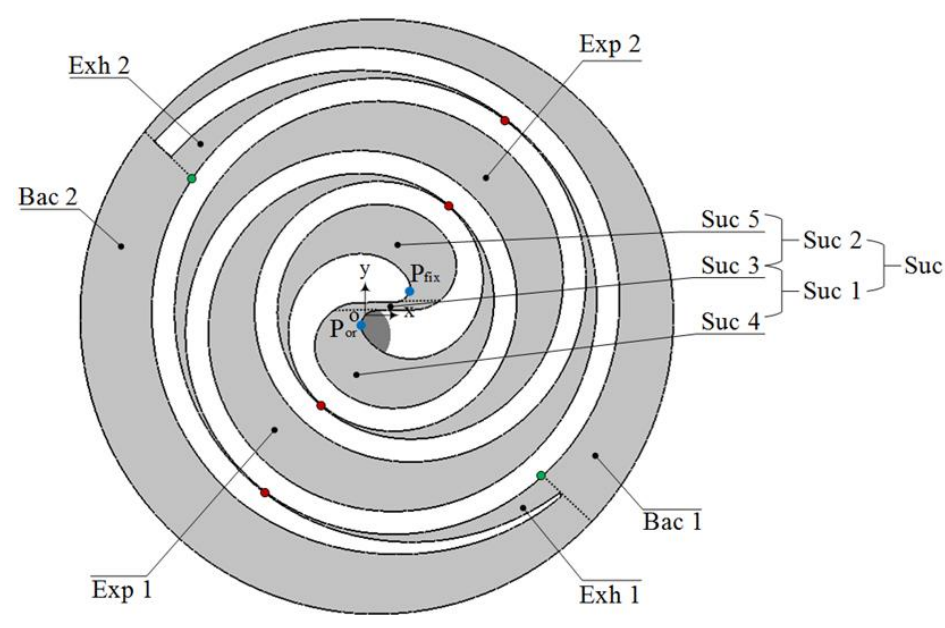

Fig. 7 Definition of various scroll working sub-chambers.

The uneven distributions of pressure in the working chambers have a significant influence on gas forces [16], particularly due the pressure differences between the adjacent chambers. In order to determine the variations of the pressure differences between the adjacent chambers, the characteristic lines along the tip of scroll profiles from the inside outwards are created, and their starting points are labeled as $\mathrm{P}_{\text {fix }}$ on the fixed scroll tip and $\mathrm{P}_{\text {or }}$ on the orbiting scroll tip respectively, as illustrated in Fig. 7.

Fig. 8 shows the pressure difference distributions at three time instants defined in Fig. 6, which may have a significant adverse impact on the maximum forces along the scroll profile unwrap length. Each highest and lowest value corresponds to a specific mesh position along the flank lines on both scrolls. The variations of the pressure difference between the adjacent chambers for $t / T$ at $13 / 15$ and 1 show the similar tendency due to their quite close time.

As shown in Fig. 8(a), the maximum pressure difference values are generated at $t / T=13 / 15$ on the fixed scroll which is marked as point 1 ', and a segment of the scroll wrap from 1' to $2^{\prime}$ is suffered approximate equivalent differential pressures which represent the differential pressures at the scroll tip between the Suc4 and Exp2 chambers. When $t / T$ equals to 1, there is 
rather high differential pressure between the chambers Suc1 and Exp2 from 2" to 3". At the time of $t / T=7 / 20$, the average pressure differences between the adjacent chambers are lower than that of other two time instants. Notably, the differential pressure from marked number 4 to the fixed scroll tail at $t / T=7 / 20$ are negative, which shows that the pressure in chamber Exh2 is lower than the pressure in chamber Bac1.

As shown in Fig.8(b), the differential pressure on the orbiting scroll wrap has also the largest value at the time of $t / T=13 / 15$. It is significant that the reverse differential pressure distributions exist between the fixed and orbiting scroll in the length ranges of 2-3, 2'- 3' and 3"- 4". The inner and outer walls in these three length ranges of the fixed scroll are suffered by the pressure from the chamber Exp2 and Exp1, respectively, but just the opposite for that of the orbiting scroll. It is also notable that the pressure in chamber Exh1 is higher than the pressure in chamber Bac2 at the time of $t / T=7 / 20$, thus the differential pressures of the fixed and orbiting scroll tails are of a similar order of magnitude but in the opposite direction.

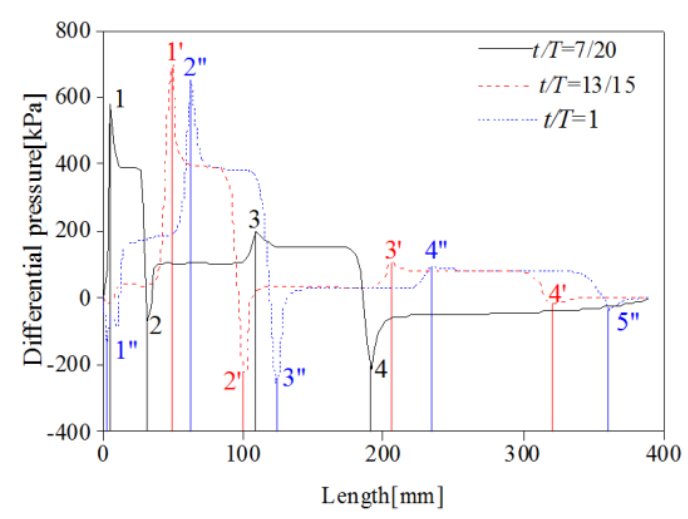

(a)

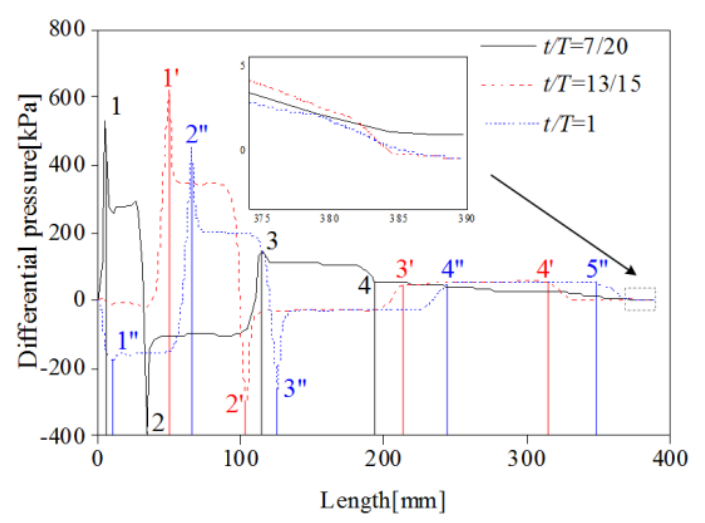

(b)

Fig. 8 Differential pressures on the scroll tips along scroll profile unwrap length: (a) fixed scroll, and (b) orbiting scroll.

As mentioned above, the differential pressure distributions at the end of the fixed and orbiting scroll at $t / T=7 / 20$ present reverse directions and differ from that at the time of 
$t / T=13 / 15$ and 1 . The pressure distributions and streamlines in the exhaust and backpressure chambers on the cross-section perpendicular to the $\mathrm{z}$-axis at a scroll height of $\mathrm{z}=16.5 \mathrm{~mm}$ at three different times are shown in Fig. 9. The pressures in chamber Exh1 are higher than that in Exh2 when $t / T$ equals to $13 / 15$ and 1, but the pressure distribution of the two chambers at $t / T=7 / 20$ indicates the opposite. As illustrated in Fig.9 (b) and (c), the gases from chamber Exh1 and Exh2 flow into the chambers Bac1 and Bac2 in form of a leakage flow through the narrow passage. The volumes of chamber Exh1 and Exh2 are both decreasing while the volumes of chamber Bac1 and Bac2 are increasing. However, the increasing rates of the two backpressure chamber volumes are different, as the volume of chamber Bac1 increases with a higher rate because of the unilateral arrangement for the outlet pipe [16]. Thus, the pressure in chamber Bac2 is much lower than in chamber Bac1 and some gas is driven back because of the pressure difference between the two backpressure chambers. As a result, a high-pressure region near the end of the orbiting wrap outer wall is formed within the constraint of the scroll expander shell. As shown in Fig.9 (a), the gas flow is driven from chamber Exh1 to chamber Bac1 by inertia with a small pressure gradient. Due to the decreasing volume of chamber Bac1 and the constraints of the shell and fixed scroll outer walls, the pressure in chamber Bac1 is relatively high. An increasing back pressure leads to a high flow resistance during the discharging process and the pressure in chamber Exh1 increases. At the same time, the low-pressure gas from chamber Exh2 is driven to chamber Bac2 by inertia and a portion of the gas flows into the outlet pipe. Furthermore, the other potion of the gas continues to flow along the wall under the inertia force and some gas flows even into chamber Bac1. The different flow patterns between the gas in the symmetric chambers Bac1 and Bac2 at $t / T=7 / 20$ 
result in the reverse differential pressures at the end of the fixed and orbiting scroll.

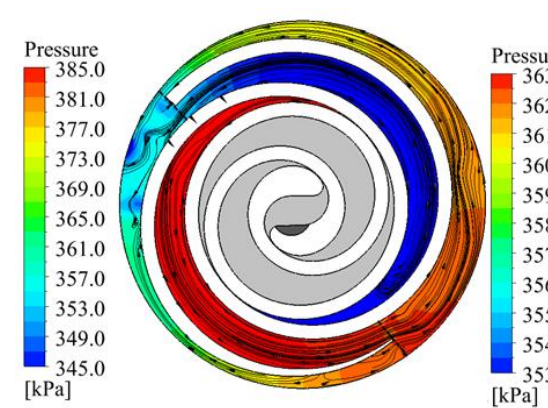

(a)

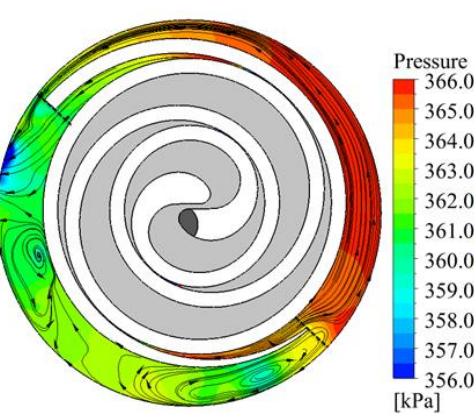

(b)

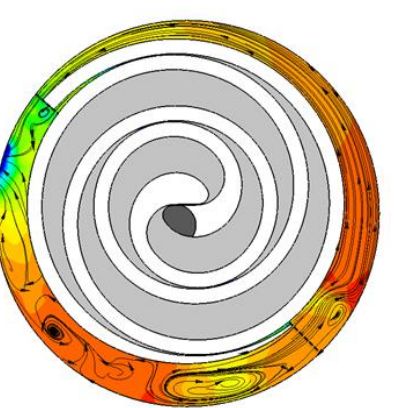

(c)

Fig. 9 Pressure distributions and streamlines in the exhaust and backpressure chambers at three different time instants: (a) $t / T=7 / 20$, (b) $t / T=13 / 15$, and (c) $t / T=1$.

3.3 Temperature distributions in the scroll chambers and scroll parts

The temperature distributions of the gas in the scroll chambers on the cross-section perpendicular to the $\mathrm{z}$-axis at a scroll height of $\mathrm{z}=3 \mathrm{~mm}$ are shown in Fig.10. The temperature in the suction chamber is highest, and the symmetrical chambers have asymmetrical temperature distributions. There is also an uneven temperature distribution in a single chamber, particularly in the crescent-shaped expansion chamber. The temperature of the gas near the wall is higher than that at the central region of the same chamber because of the heating effect from the scroll lateral wall. Moreover, the temperature gradient of the downstream gas is larger than that of the upstream gas in the same chamber, while the gas temperature in downstream area is lower compared to the upstream gas. It is because of the orbiting scroll motion that results in the squish action of the upstream gases, thus the internal energy of the upstream gas increases. Meanwhile, the downstream gases are extracted with expansion effects. 


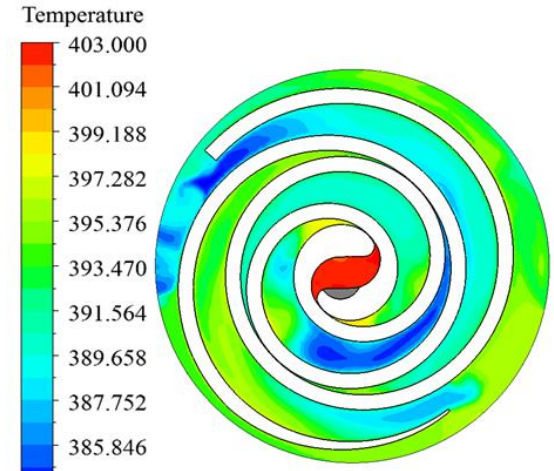

$[\mathrm{K}]$

(a)
383.940

Fig. 10

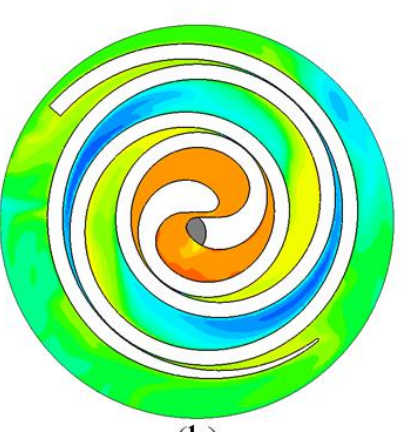

(b)

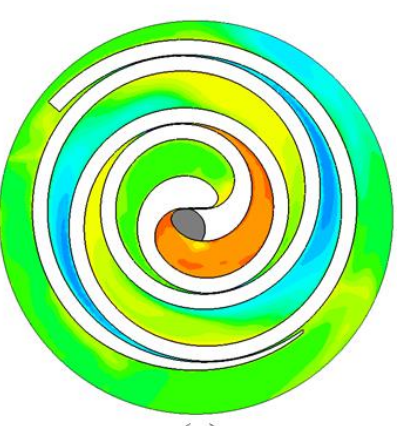

(c)

Fig. 10 Temperature distributions in the scroll chambers at three time instants: (a) $t / T=7 / 20$, (b)

$$
t / T=13 / 15 \text {, and (c) } t / T=1 \text {. }
$$

As is shown in Fig. 11, the gas enters into the inlet pipe and continuously flows along the circumferential direction in the suction plenum, when the gas flows close to the central regions of the plenum, a large-scale swirl is generated near the suction port and the temperature of the gas near the central regions of the plenum decreases significantly. Therefore, the thermal energy in the suction plenum can be transferred to the scrolls, particularly in the outer region of the scroll wraps. The back wall of the fixed scroll endplate is heating by the gas in the suction plenum, and it is one of the reasons why the differences between the temperature distributions of the scroll parts.

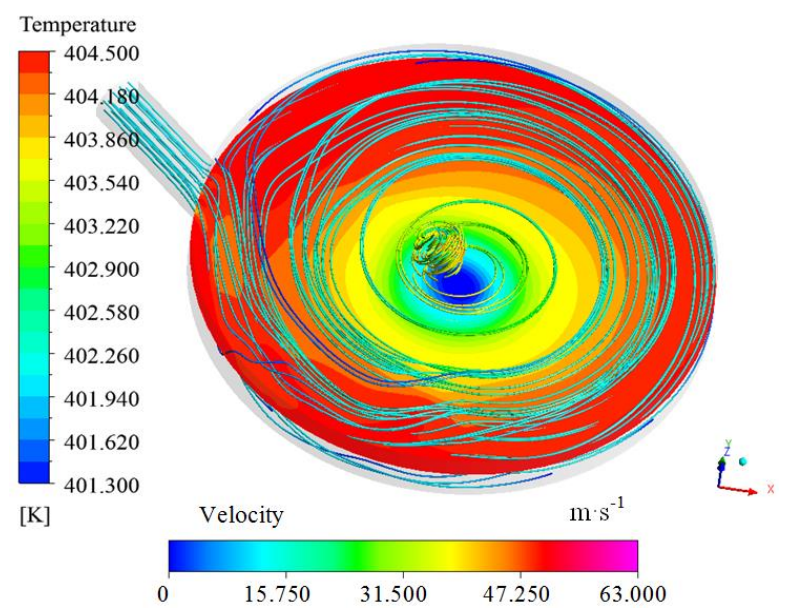

Fig.11 Fluid temperature distribution on the middle cross section and 3D streamlines in the suction 


$$
\text { plenum at } t / T=7 / 20
$$

Fig. 12 shows the temperature distributions of the scroll wraps when $t / T=7 / 20$. Due to the thermal inertia of the solid, the temperature distributions of the solid vary little over time when the expander works under the steady-state operating point[8]. Song et al. [16] indicated that when the swirl flow entered the suction chamber through the suction port, the energy diffusion mode of the gas flow changed with the blocking effect of the orbiting top scroll, due to the volume and shape variations of the suction chamber. Moreover, a high velocity gradient and different scale vortices are present during the whole suction process. Thus, the convection heat transfer between the walls and the gas is enhanced and dominates the temperature of the top scroll. Similarly, because of the enhanced convective heat transfer between the scroll wrap tails and the gas during the discharging process, the temperature of the scroll tails declined significantly. The variation range of temperature distribution in the scroll bodies is within $7 \mathrm{~K}$. However, the temperature distributions of the scrolls are not only influenced by convective heat transfer between walls and gas, but also heat transfer from the gas inside the suction plenum to the scroll endplate. There is a slight increase of both scrolls temperature in the marked region of Fig. 12, and is a consequence of the maximum heat flow from the suction plenum to the scrolls.

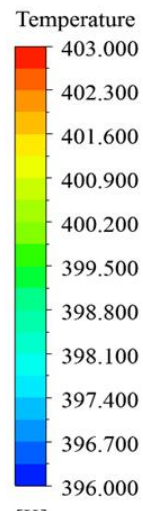

$[\mathrm{K}]$

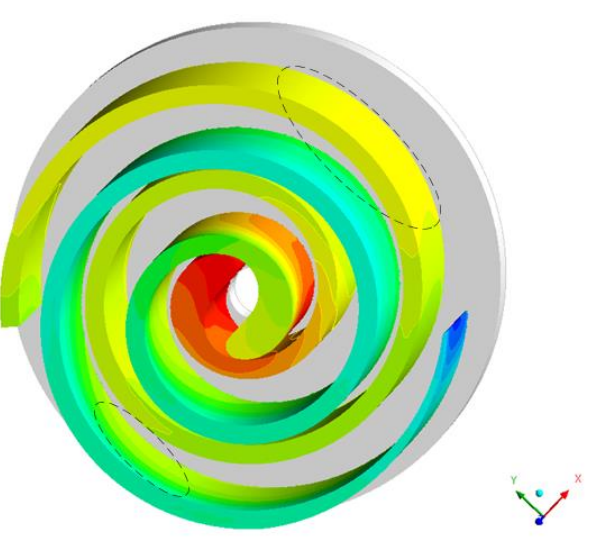

20 
Fig. 12 Temperature distributions on the scroll parts at $t / T=7 / 20$

\section{Deformation analysis of the scroll expander}

\subsection{Deformations under thermal loads}

As mentioned in section 3, there are three time instants which may have a significant adverse impact on the maximum gas forces. Hence, the most critical time when the maximum deformation of the scroll wraps takes place is required to be determined for further analysis.

Fig.13 shows the thermal deformation of the fixed and orbiting scroll at $t / T=7 / 20$. The maximum deformation of the fixed scroll with a value of $65.505 \mu \mathrm{m}$ occurs at the tip of the top scroll. The location of the maximum thermal deformation of the orbiting scroll, as shown in Fig.13(b), occurs at tip of the outer wrap of the orbiting scroll, where it is close to the scroll tail. Although the temperature gradient within the orbiting scroll is higher than that within the fixed scroll, the maximum deformation value of the orbiting scroll is much lower than that of the fixed scroll due to the axial restraint of the thrust ball bearing on the backside of the orbiting scroll endplate. The maximum thermal deformation of the other time instants present similar trend and values, as shown in Table 4. The thermal deformations of both scrolls are large and only change slightly over the time, because the temperature variations are minimal as described in section 3.3.

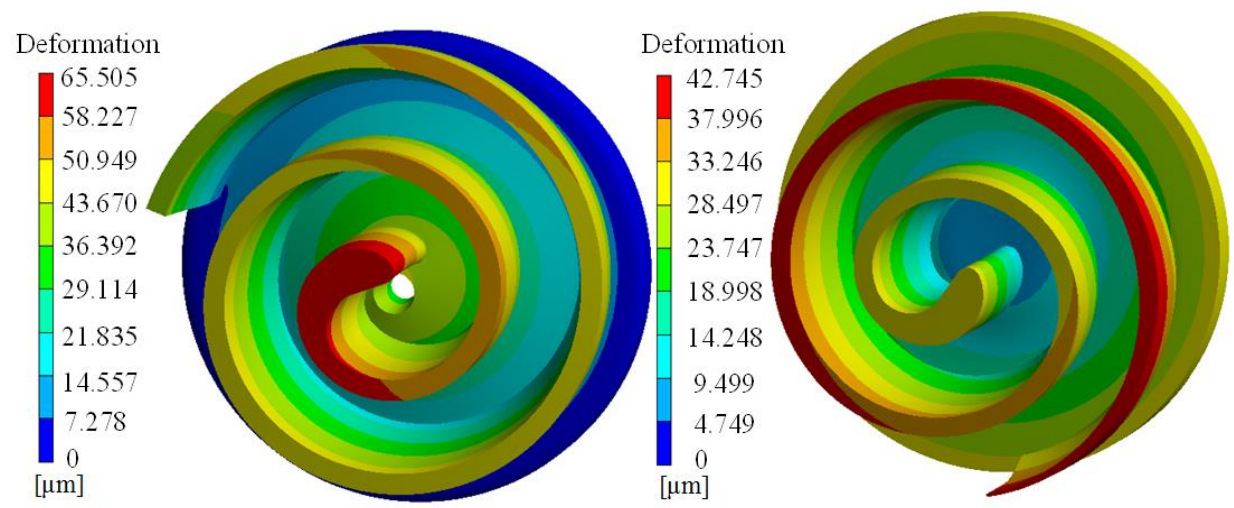


(a)

Fig. 13 Thermal deformation distributions at $t / T=7 / 20$ : (a) fixed scroll, and (b) orbiting scroll.

Table 4 Simulation results of thermal deformation.

\begin{tabular}{lccc}
\hline Parameters & $t / T=7 / 20$ & $t / T=13 / 15$ & $t / T=1$ \\
\hline $\begin{array}{l}\text { Maximum deformation of the fixed scroll }[\mu \mathrm{m}] \\
\text { Location of the maximum deformation of the fixed }\end{array}$ & 65.505 & 65.444 & 65.434 \\
$\begin{array}{l}\text { scroll[-] } \\
\text { Maximum deformation of the orbiting scroll }[\mu \mathrm{m}]\end{array}$ & 42.745 & 42.707 & 42.678 \\
$\begin{array}{l}\text { Location of the maximum deformation of the } \\
\text { orbiting scroll[-] }\end{array}$ & The tip of the outer wrap near the scroll tail \\
\hline
\end{tabular}

4.2 Deformations under pressure loads

The deformation distributions of both scrolls caused by pressure loads at $t / T=7 / 20$ are shown in Fig.14. The maximum pressure deformation of the fixed scroll wrap is nearly $29 \mu \mathrm{m}$. The supporting effect on the central region of the fixed scroll endplate is reduced due to the arrangement of the suction port, which leads to a more significant deformation along the axial direction than that along the radial direction, as shown in Fig.14(a). Consequently, the backpressure in the suction plenum which is exerted on the backside of the fixed scroll endplate which cause the two scrolls to contact with each other. Furthermore, the deformations of the orbiting scroll are primarily distributed along the radial direction of the orbiting scroll tail, as illustrated in Fig.14(b), because the modification of the scroll profile reduces the thickness of the scroll tail. 


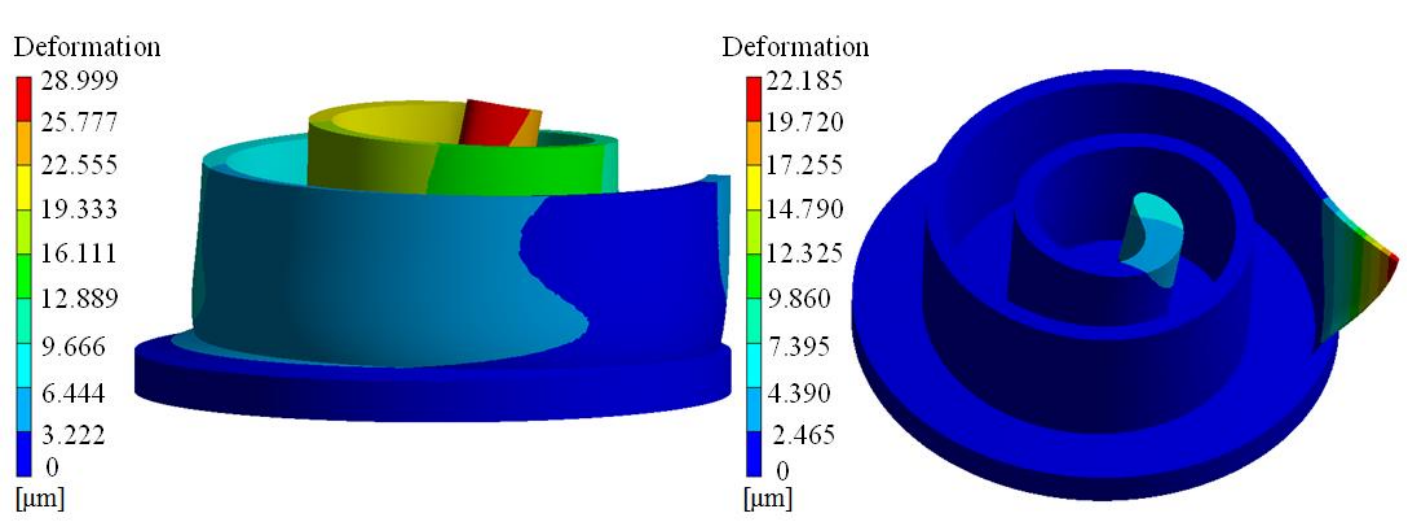

(a)

(b)

Fig. 14 Pressure deformation distributions at $t / T=7 / 20$ : (a) fixed scroll, and (b) orbiting scroll.

Compared with $t / T=7 / 20$, the maximum pressure deformation of the other time instants presents the same location but with different magnitudes, as shown in Table 5. For the orbiting scroll, the direction of the maximum pressure deformation at $t / T=7 / 20$ differs from those of the other two time instants. As already presented in Fig.8(b), the differential pressure at the tip of the orbiting scroll tail at $t / T=7 / 20$ is opposed to those at the other two time instants. It follows that the maximum deformation of the orbiting scroll at $t / T=7 / 20$ is more than twice as high than that of the other two moments while still acting in the opposite direction.

Table 5 Simulation results of pressure deformation.

\begin{tabular}{lccc}
\hline Parameters & $t / T=7 / 20$ & $t / T=13 / 15$ & $t / T=1$ \\
\hline $\begin{array}{l}\text { Maximum deformation of the fixed scroll }[\mu \mathrm{m}] \\
\text { Location of the maximum deformation of the fixed }\end{array}$ & 28.999 & 23.490 & 22.694 \\
scroll[-] & The tip of the top scroll near the suction port \\
Maximum deformation of the orbiting scroll $[\mu \mathrm{m}]$ & 22.185 & 7.595 & 6.331 \\
$\begin{array}{l}\text { Location of the maximum deformation of the } \\
\text { orbiting scroll[-] }\end{array}$ & & The tip of the scroll tail \\
\hline
\end{tabular}

\subsection{Deformations under inertial load}

Fig. 15 presents where the maximum deformation induced by inertial load occurs and the deformation distribution at $t / T=7 / 20$. The deformations only occur at the end area of the scroll 
and lead to maximum deformations at the tip of the scroll tail. The similar deformation distribution and region of the maximum deformation are showed in Table 6 at $t / T=13 / 15$ and $t / T=1$.

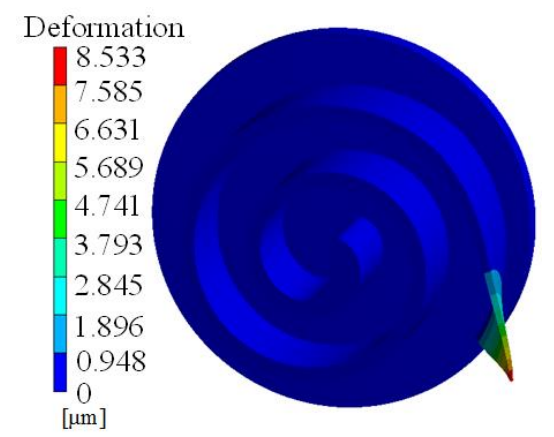

Fig. 15 Deformation distributions induced by inertial load at $t / T=7 / 20$.

Table 6 Simulation results of deformation under inertial load.

\begin{tabular}{lccc}
\hline Parameters & $t / T=7 / 20$ & $t / T=13 / 15$ & $t / T=1$ \\
\hline Maximum deformation $[\mu \mathrm{m}]$ & 8.553 & 9.193 & 9.821 \\
Location of the maximum deformation $[-]$ & & The tip of the scroll tail & \\
\hline
\end{tabular}

4.5 Deformations under coupling action

Fig.16 shows that the deformations of the scroll parts under the effect of muti-fields coupling at $t / T=7 / 20$. The maximum deformation value of the fixed scroll is $92.150 \mu \mathrm{m}$ and the value of the orbiting scroll is $69.735 \mu \mathrm{m}$. Table 7 lists the simulation results of the deformations under coupling action at three different time instants. For the fixed scroll, the region of the largest deformation all occurs at the tip of the top scroll. The maximum deformation of the orbiting scroll is considerably similar to the thermal deformation, it is also much more affected by the pressure distribution. When $t / T$ equals to $13 / 15$ or 1 , the pressure deformations at the tip of the scroll tail run in the opposite direction compared to that of the thermal deformations, as mentioned in section 4.1 and 4.2. The thermal and pressure deformations partially cancel each other out; therefore, their coupling deformation values are much lower than the deformation at $t / T=7 / 20$. Both the largest deformations of the fixed and 
orbiting scroll occur at $t / T$ equals to $7 / 20$. Hence, this is the critical and detrimental time when the largest axial gas force exerted on the fixed scroll endplate.

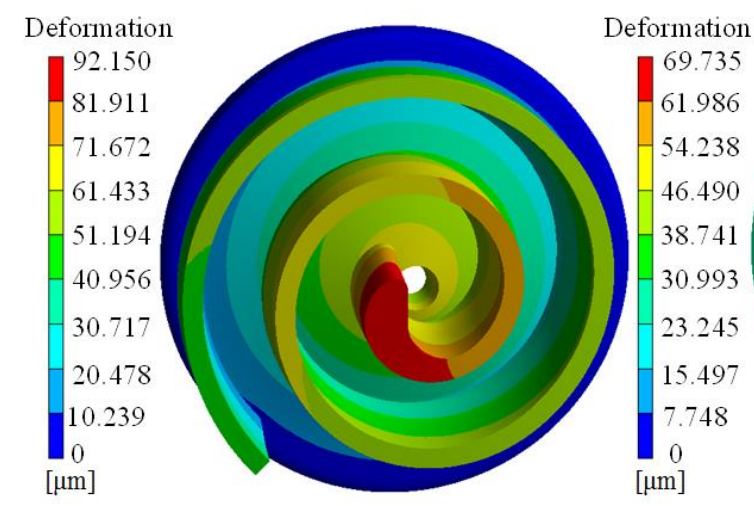

(a)

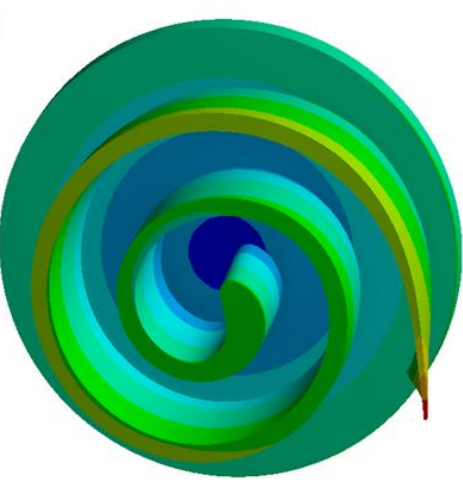

(b)

Fig. 16 Deformation distributions under coupling action at $t / T=7 / 20$ : (a) fixed scroll, and (b) orbiting scroll.

Table 7 Simulation results of coupling deformation.

\begin{tabular}{lccc}
\hline Parameters & $t / T=7 / 20$ & $t / T=13 / 15$ & $t / T=1$ \\
\hline $\begin{array}{l}\text { Maximum deformation of the fixed scroll }[\mu \mathrm{m}] \\
\begin{array}{l}\text { Location of the maximum deformation of the fixed } \\
\text { scroll[-] }\end{array}\end{array}$ & 92.150 & 87.226 & 87.129 \\
$\begin{array}{l}\text { Maximum deformation of the orbiting scroll }[\mu \mathrm{m}] \\
\text { Location of the maximum deformation of the } \\
\text { orbiting scroll[-] }\end{array}$ & 69.735 & 44.910 & 44.386 \\
\hline
\end{tabular}

\section{Conclusions}

In this research, a one-way fluid-thermal-solid coupling model for a scroll expander was developed by coupling CFD and FEA modeling techniques. Temperature and pressure distributions as a function of time were calculated and analysed in details, and the deformation under different loads were investigated in details. The following conclusions can be drawn from this work:

(1) Three time instants which may have a significant adverse impact on the maximum forces are selected to determine the most critical time for the occurrence of the 
maximum deformation of the scroll wraps. The differential pressure distributions at the end of the fixed and orbiting scroll at $t / T=7 / 20$ is reverse compared with those of the other two instants due to the different flow patterns between the gas in the two backpressure chambers.

(2) The heating action of the gas in the suction plenum plays a prominent role in the temperature distribution of the solid. The temperature along the trajectory of the scroll profiles decrease at first, but then increase, before the temperature finally shows a significant decaying trend rather than a steady decrease from the inside outwards.

(3) The maximum inertial force deformation is small and appears at the orbiting scroll tail. The directions of the pressure deformation are reverse within the same locations at different times due to the variations of the differential pressure. When the deformations induced by pressure loads have the same direction with that of the thermal loads, there is an overlap between the two deformations resulting in an increase of the coupling deformation. On the contrary, these can also offset each other if the deformations induced by pressure loads have the opposite direction compared to that of thermal loads and which in turn lead to a decrease of the coupling deformation.

(4) The deformations induced by pressure loads are smaller than that by thermal loads. Hence, the distribution characteristics of the deformation under coupling action are similar to that of the thermal deformation, while the deformations induced by pressure loads have only a slight influence on the distribution characteristics of the total deformation and only change the values. The largest deformations of the fixed and orbiting scroll occur at $t / T$ equals to $7 / 20$ with values of $92.15 \mu \mathrm{m}$ and $69.735 \mu \mathrm{m}$ 
respectively, so the critical and detrimental time is the instant of the largest axial gas force exerted on the fixed scroll endplate. 


\section{Nomenclature}

$F_{r} \quad$ Radial force parallel to orbiting radius (N)

$F_{t} \quad$ Tangential force perpendicular to orbiting radius (N)

$F_{a} \quad$ Axial force perpendicular to the surface of orbiting scroll endplate (N)

$F_{o c} \quad$ Centrifugal force exerted on orbiting scroll (N)

$h \quad$ Enthalpy $(\mathrm{J} / \mathrm{mol})$

I Current (A)

$K_{\text {eff }}$ Thermal conductivity of the solid $(\mathrm{W} /(\mathrm{m} \cdot \mathrm{K}))$

$M_{d} \quad$ Driving gas moment $(\mathrm{N} \mathrm{m})$

$m_{o} \quad$ Mass of orbiting scroll $(\mathrm{kg})$

$N$ Count of cells (-)

$n$ Rotating speed ( $\mathrm{r} / \mathrm{min})$

$P_{i} \quad$ Inlet pressure $(\mathrm{kPa})$

$P_{o} \quad$ Outlet pressure $(\mathrm{kPa})$

$q$ Heat flux $\left(\mathrm{W} / \mathrm{m}^{2}\right)$

$R_{o r} \quad$ Orbiting radius of orbiting scroll (m)

$t$ Time (s)

$T_{i} \quad$ Inlet temperature (K)

$T$ Time period (s)

$U$ Voltage (V)

$\stackrel{\perp}{V}$ Solid velocity vector $(\mathrm{m} / \mathrm{s})$

$W$ Output power $(\mathrm{kW})$

Greek symbols

$\rho \quad$ Density $\left(\mathrm{kg} / \mathrm{m}^{3}\right)$

$\eta \quad$ Expander efficiency (-)

$\omega$ Angular velocity ( $\mathrm{rad} / \mathrm{s})$

Subscripts

bot bottom surface

exp experiment

$\mathrm{f}$ fluid

fix fixed scroll

$i$ surface element number

or orbiting scroll

ple suction plenum

rad radiation

s solid

sim simulation

tip scroll tip

top top surface

w wall

Acronyms

CFD Computational Fluid Dynamics

FEM Finite Element Methods

NIST National Institute of Standards and Technology

ORC Organic Rankine Cycles

WHR Waste Heat Recovery 


\section{References}

[1] V. Lemort, S. Quoilin, C. Cuevas, J. Lebrun, Testing and modeling a scroll expander integrated into an Organic Rankine Cycle. Applied Thermal Engineering, 29(2009) 3094-3102.

[2] S. Declaye, S. Quoilin, L Guillaume, V. Lemort, Experimental study on an open-drive scroll expander integrated into an ORC (organic rankine cycle) system with R245fa as working fluid. Energy, 55(2013) 173-183.

[3] P. Song, M. Wei, L. Shi, S.N. Danish, C. Ma, A review of scroll expanders for organic rankine cycle systems, Applied Thermal Engineering, 75(2015) 54-64.

[4] M. Morini, C. Pavan, M. Pinelli, A. Suman, Analysis of a scroll machine for micro ORC applications by means of a RE/CFD methodology. Applied Thermal Engineering, 80(2015) 132-140.

[5] K. Suefuji, M. Shiibayshi, R. Minakate, K. Tojo, Deformation analysis of scroll members in hermetic scroll compressors for air conditioners. Proceedings of International Compressor Engineering Conference at Purdue, (1988)583-589.

[6] D. Yu, T. Ameel, R. Warrington, Thermal and static finite element analysis of fixed scroll deformation, Proceedings of International Compressor Engineering Conference at Purdue, (1996) 465-470.

[7] J.M. O'Leary, T.J. Weadock, G.W. Gatecliff, Scroll Compressor Cupping Analysis. Proceedings of International Compressor Engineering Conference at Purdue, (1998) 613-617.

[8] C. Lin, Y. Chang, K. Liang, C. Hung, Temperature and thermal deformation analysis on scrolls of scroll compressor. Applied Thermal Engineering, 25(2005) 1724-1739.

[9] Y. Liu, Y. Tang, Y. Chang, Y. Yang, Optimum design of scroll profiles created from involute of circle with variable radii by using finite element analysis. Mechanism and Machine Theory, 55(2012) 1-17.

[10] M. Cui, Numerical study of unsteady flows in a scroll compressor. Fluids engineering, 128 (2006) 947-955.

[11] L. Pereira, J. Deschamps, A numerical study of convective heat transfer in the compression chambers of scroll compressors. Proceedings of International Compressor Engineering Conference at Purdue, (2012)2191.

[12] K. Ooi, J. Zhu, Convective heat transfer in a scroll compressor chamber: a 2-D simulation. Thermal Sciences, 43(2004) 677-688.

[13] P. Song, M. Wei, L. Shi, C. Ma, Numerical simulation of three-dimensional unsteady flow in a scroll expander applied in waste heat recovery. International Conference on Pumps and Fans with Compressors and Wind Turbines, 52 (2013).

[14] J. Chang, C. Chang, T. Hung, J. Lin, K. Huang. Experimental study and CFD approach for scroll type expander used in low-temperature organic Rankine cycle. Applied Thermal Engineering, 73 (2014) 1444-1452.

[15] M. Wei, P. Song, B. Zhao, L. Shi, Z. Wang, C. Ma, Unsteady flow in the suction process of a scroll expander for an ORC waste heat recovery system. Applied Thermal Engineering, 78(2015) 460-470.

[16] P. Song, M. Wei, Z. Liu, Effects of suction port arrangements on a scroll expander for a small scale ORC system based on CFD approach. Applied Energy, 150 (2015)274-285.

[17] Z. Vadim, B. Nikolai, G. Michael, Chapter five-Industrial Casting Aluminum Alloys. In Casting Aluminum Alloys, Elsevier, (2007)327-396. 
[18] Online Materials Information Resource - MatWeb, http://www.matweb.com/search/SpecificMaterial.asp?bassnum=MCFE14.

[19] E. Morishita, M. Sugihara, T. Inaba, T. Nakamura, Scroll compressor analytical model. Proceedings of International Compressor Engineering Conference at Purdue, (1984) 487-495 [20] H. Bauer, Bosch automotive handbook 8th edition, Stuttart: Robert Bosch GmbH, 2011. [21] S. Quoilin, S. Declaye, B. F. Tchanche, V. Lemort, Thermo-economic optimization of waste heat recovery Organic Rankine Cycles, Applied Thermal Engineering, 31(2011)2885-2893. 